\title{
Superconductivity in doped polyethylene at high pressure
}

\author{
José A. Flores-Livas, ${ }^{1}$ Migle Graužinyte,,${ }^{1}$ Lilia Beori, ${ }^{2}$ Gianni Profeta, ${ }^{3}$ and Antonio Sanna ${ }^{4}$ \\ ${ }^{1}$ Department of Physics, Universität Basel, Klingelbergstr. 82, 4056 Basel, Switzerland \\ ${ }^{2}$ Department of Physics, Sapienza Universita' di Roma, Italy \\ ${ }^{3}$ Dipartimento di Fisica Università degli Studi di L'Aquila and SPIN-CNR, I-67100 L'Aquila, Italy \\ ${ }^{4}$ Max-Planck Institut für Microstrukture Physics, Weinberg 2, 06120 Halle, Germany
}

\begin{abstract}
In this work we study the pressure-dependent phase diagram of polyethylene $\left(\mathrm{H}_{2} \mathrm{C}\right)_{x}$ from 50 up to $200 \mathrm{GPa}$. Low-symmetry, organic polymeric phases, that are dynamically stable and thermodynamically competitive with elemental decomposition, are reported. Electronic structure calculations reveal that the band gap of the lowest energy polymeric phase decreases from $5.5 \mathrm{eV}$ to $4.5 \mathrm{eV}$ in the 50 - $200 \mathrm{GPa}$ range, but metalization occurs only for pressures well above $500 \mathrm{GPa}$. The possibility of metalization via doping was also investigated, observing that it can be achieved through boron substitution at carbon sites. We report a sizable electron-phonon coupling $(\lambda \simeq 0.79)$ in this metallic phase, with an estimated superconducting transition temperature of about $35 \mathrm{~K}$. However, a rather narrow domain of stability is found; most of the dopant elements render the polymeric phases unstable and induce amorphization. This suggests that doping under pressure, though presenting an alternative route to find high temperature superconductors, would be challenging to achieve experimentally.
\end{abstract}

\section{INTRODUCTION}

High pressure is an exciting field that has evolved incredibly far since the pioneering work of Cailletet, Amagat and Bridgman ${ }^{1}$. Back in those days the maximum pressure that could be applied to materials was merely one giga-Pascal (GPa), and only primitive characterization techniques were available. Nowadays, the record pressure measures well above $500 \mathrm{GPa}$ and an entire array of tools and spectroscopies are at hand to characterize in situ the response of materials under pressure ${ }^{2,3}$.

A substantial amount of research in the field of high pressure (post-Bridgman era) was triggered by the tantalizing idea of metalizing hydrogen (Wigner-Huntington ${ }^{4}$ transition) which dates back to the mid 30's. The metalization of hydrogen is seen as the holy-grail of high pressure research, it has been a compelling subject of great interest for many scientists ranging from experimental chemists and physicists to theoreticians, including Prof. Gross. However, despite the great advancement of high pressure techniques and tools, the metalization of hydrogen has been elusive.

The landscape in the high-pressure field changed drastically in 2004, when in his pioneering work Ashcroft suggested chemically precompressed materials as an alternative route to decrease the tremendous pressure necessary to metalize hydrogen ${ }^{5}$. Eremets et al. ${ }^{6}$ successfully proved the principle in 2008 by metalizing silane $\left(\mathrm{SiH}_{2}\right)$. Although subsequent studies attributed these results to probable decomposition or the existence of other stoichiometries $^{7-12}$, this work remains the first solid evidence of chemically precompressed materials at high pressure.

More recently the prediction of stable crystals of $\mathrm{H}_{3} \mathrm{~S}^{13}$ and their subsequent discovery by Drozdov-Eremets ${ }^{14}$ in 2015 broke all previous critical transition temperature $\left(\mathrm{T}_{\mathrm{C}}\right)$ records by requiring a mere $-73^{\circ} \mathrm{C}(\sim 200 \mathrm{~K})$ for the onset of superconductivity. Although, the stable compositions and the superconducting properties were predicted prior to the experiments, it is worth mentioning that the synthesis was carried out independently, i.e. without the knowledge of the predictions of the stable stoichiometries.

Sulfur-hydride is not an isolated example of conventional high- $\mathrm{T}_{\mathrm{C}}$ superconductivity at high pressures ${ }^{15-19}$, $\mathrm{H}_{3} \mathrm{Se}^{20,21}$, and phosphine $\left(\mathrm{PH}_{3}\right)$ has been observed to superconduct at transition temperatures as high as $100 \mathrm{~K}$ at $200 \mathrm{GPa}^{22,23}$. Many other systems have been theoretically proposed based on superhydrides reviving interest also in structural transformations of individual elements and their tendency towards metallic structures $^{24-28}$. Many - if not all - of the structures under high pressure (HP) are metastable phases ${ }^{29}$ (i.e. out of thermodynamic equilibrium). Consequently, many of the HP phases cannot be recovered to ambient conditions. This translates to one of the greatest challenges of using high pressure in practical applications ${ }^{30}$. Different strategies to aid stabilization of HP-materials have been proposed, where a major role is played by the precise control of thermodynamic conditions ${ }^{25,28,31}$.

The lightest of all the elements deserves its own chapter. As mentioned before, hydrogen is predicted to become metallic under high pressure ${ }^{32}$, and is arguably the best candidate for the first place in the race for room temperature superconductivity ${ }^{33}$. Its low mass results in high-phonon frequencies and combined with the lack of core electrons, that enables strong electron-phonon coupling to arise when covalent bonds are compressed ${ }^{34,35}$. However, hydrogen remains insulating at least up to 350 $\mathrm{GPa}^{36-39}$. Indeed, it is a heated debate ${ }^{40}$ whether the metalization can be reached at $400 \mathrm{GPa}^{41}$ or higher pressures are necessary ${ }^{42,43}$.

On the other hand, carbon - one of the hardest materials known to date - adopts the notorious $F d 3 m$ cubic crystal structure of diamond at high pressure. Despite the variations in its electronic band gap ${ }^{44,45}$, the structure remains insulting across the entire pressure range at 


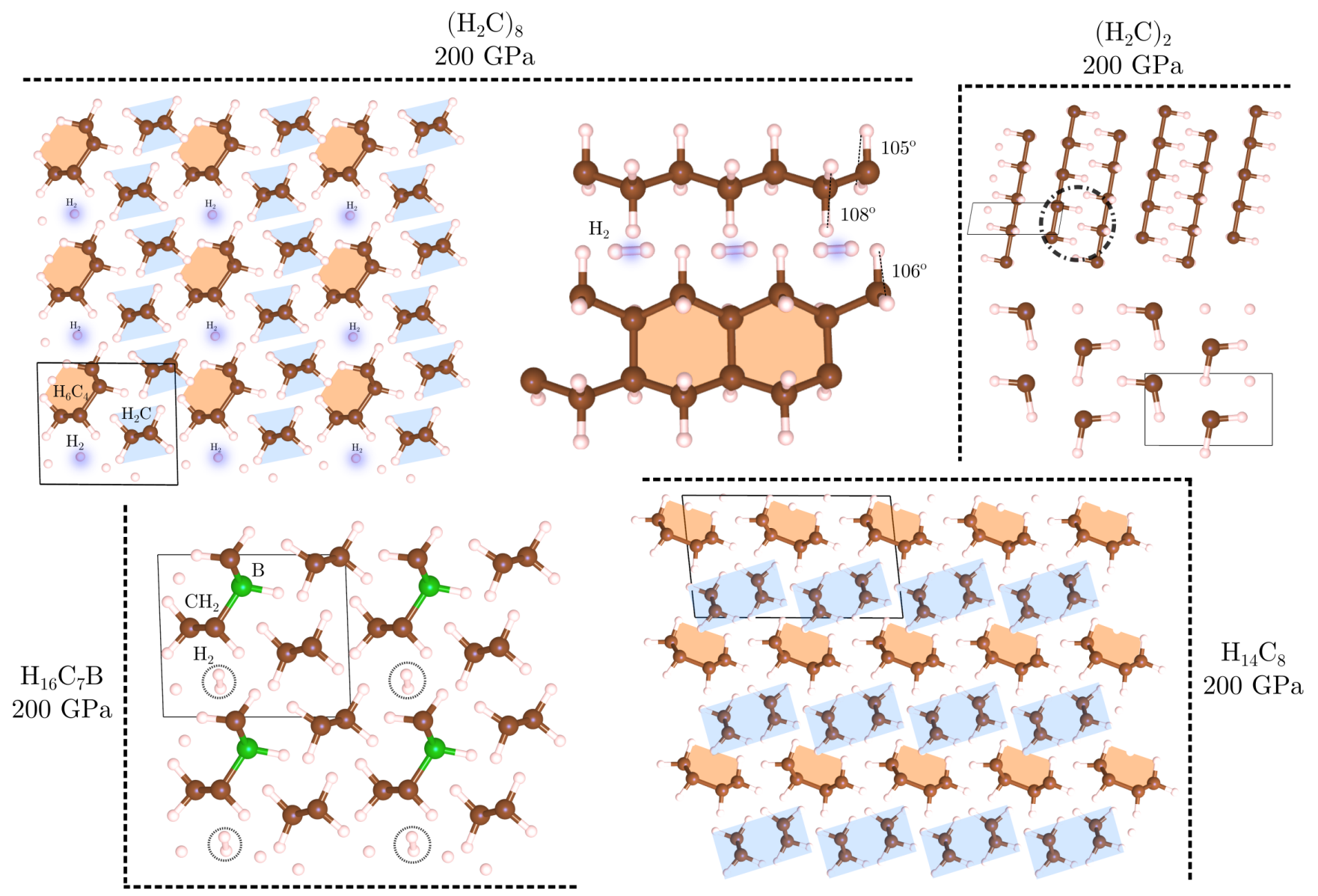

Figure 1. Polymeric phases of polyethylene at high pressure. All structures have P1 symmetry. Top left: two distinct polymeric lines depicted in orange $\left(\mathrm{H}_{6} \mathrm{C}_{4}\right)$ and blue $\left(\mathrm{H}_{2} \mathrm{C}\right)$ and $\mathrm{H}_{2}$ molecules. Bottom left: doped phase of polyethylene with $12 \%$ boron. Right bottom: two distinct polymeric lines of $\mathrm{H}_{2} \mathrm{C}$ are depicted in yellow and blue without $\mathrm{H}_{2}$ components.

least up to $500 \mathrm{GPa}$. It is indeed, thanks to this material that the high pressure field has flourished ${ }^{46}$ : diamond anvil cells (DAC) allow for controlled experiments reaching static pressures equivalent to those at the Earth's core $\sim 400 \mathrm{GPa}$ (for comparison, $1 \mathrm{GPa}$ is equivalent to 10,000 bar that is equivalent to 9,869 atmospheres).

It is well understood, that compression of molecular systems at high pressure increases the electron-orbital overlap between neighboring atoms resulting in an increase of the band dispersions consequently closing the electronic band gap. Chemical precompression ${ }^{5}$ is certainly one promising route to reduce the metalization pressure on insulating elements ${ }^{47}$, but not the only one. Another method to reach metalization is chemical doping under pressure - a path previously used at ambient pressures to render standard insulators superconducting ${ }^{48-51}$. This approach was demonstrated for $\mathrm{H}_{2} \mathrm{O}$, one of the most abundant and well-studied substances, theoretically showing that when doped with nitrogen via substitutional doping water could become superconducting with transition temperatures as high as $60 \mathrm{~K}$. This represents a promising avenue that could be explored for high- $\mathrm{T}_{\mathrm{C}}$ candidates ${ }^{52}$. Thus, an entirely new playground emerges, in which pressure, metastability and controlled doping play a crucial, yet intertwined role, the latter being the most challenging and complex to achieve experimentally. As a matter of fact, doping is a complex procedure that not only relies on the mere statics of the chemical system, but also the complex dynamics of experimental synthesis. However, we motivate the field by noting that in many classes of materials, such as pnictides $^{53,54}$, layered materials ${ }^{55,56}$ and unconventional superconductors, most of the interesting phenomena only occurs at precise doping levels or through tiny structural changes ${ }^{57,58}$. As such, doping under pressure provides a novel axis for exploratory high pressure experiments, that in the near future are likely to lead to new high$\mathrm{T}_{\mathrm{C}}$ candidates and among them, hopefully, a room temperature superconductor.

All of the above fields, superconductivity under pressure $^{27,59}$, metallization and superconductivity in hydrogen $^{33}$, chemically precompressed hydrides ${ }^{20,23}$, layered materials and phase transformations under pressure ${ }^{60,61}$, have been of great interest to Prof. Gross throughout the years. Well before the discovery of $\mathrm{H}_{2} \mathrm{~S}$, he pointed us to the potential search space in this field. We freshly 


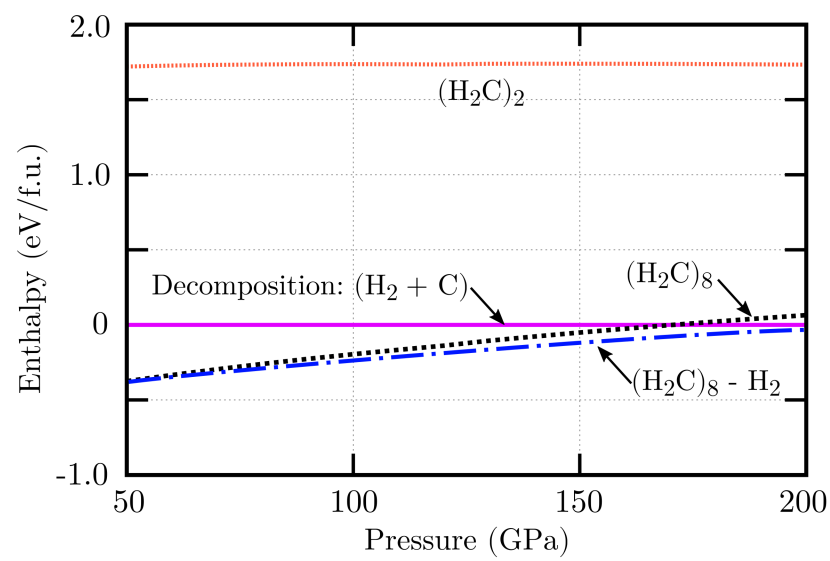

Figure 2. Calculated formation enthalpy for $\mathrm{H}_{2} \mathrm{C}$ as function of pressure towards elemental decomposition $\left(\mathrm{H}_{2}+\mathrm{C}\right)$. Small simulation cells of $\left(\mathrm{H}_{2} \mathrm{C}\right)_{2}$ are thermodynamically unstable in the pressure range studied. Large simulation cells $\left(\mathrm{H}_{2} \mathrm{C}\right)_{8}$ are considerable more stable towards decomposition.

remember the day after the $\mathrm{H}_{2} \mathrm{~S}\left(\mathrm{~T}_{\mathrm{C}}=200 \mathrm{~K}\right)$ news appeared in $\operatorname{ArXiv}$; the day after we had a meeting to discuss these results and decide on several lines of exploration. Among other systems and compositions, notably $\mathrm{H}_{2} \mathrm{Se}$ and $\mathrm{H}_{2} \mathrm{O}$, were discussed in the meeting. Although a mentor only for a short period, Prof. Gross provided me (J.A.F.-L.) with the opportunity to share great moments in Halle and to climb a steep learning curve while hosted in his group. We would like to dedicate this article to Prof. Gross, - Hardy - for his 65 th birthday.

This work investigates the structural stability of polyethylene $\left(\mathrm{H}_{2} \mathrm{C}\right)_{n}$ under pressure. The questions we want to address in this work were: is there a stable polyethylene phase under pressure that can be doped? if yes, is it superconducting? The work is organized as follows, the first section encapsulates the results of crystal structure exploration and phase stability. Subsequent sections follow the natural progression of discussing electronic properties, vibrational properties and analysis of potential dopants.

\section{CRYSTAL STRUCTURE AND STABILITY AT HIGH PRESSURE}

The experimental evidence suggest that benzene $\left(\mathrm{H}_{6} \mathrm{C}_{6}\right)_{n}$ decompose for pressures above $\sim 50 \mathrm{GPa}^{62,63}$. In fact it was demonstrated that for benzene the transformation of crystalline to an amorphous hydrogenated carbon the intermolecular $\mathrm{C}-\mathrm{C}$ distance is always the same $(\sim 2.6 \AA)^{64}$. This can also be seen as the fact that molecular systems are unstable with respect to saturated, four coordinated carbons at high pressure. Therefore, under high compression, independently of benzene or ethylene groups the formation of polymeric phases seems unavoidable $^{65,66}$. We jump-start the work by compressing
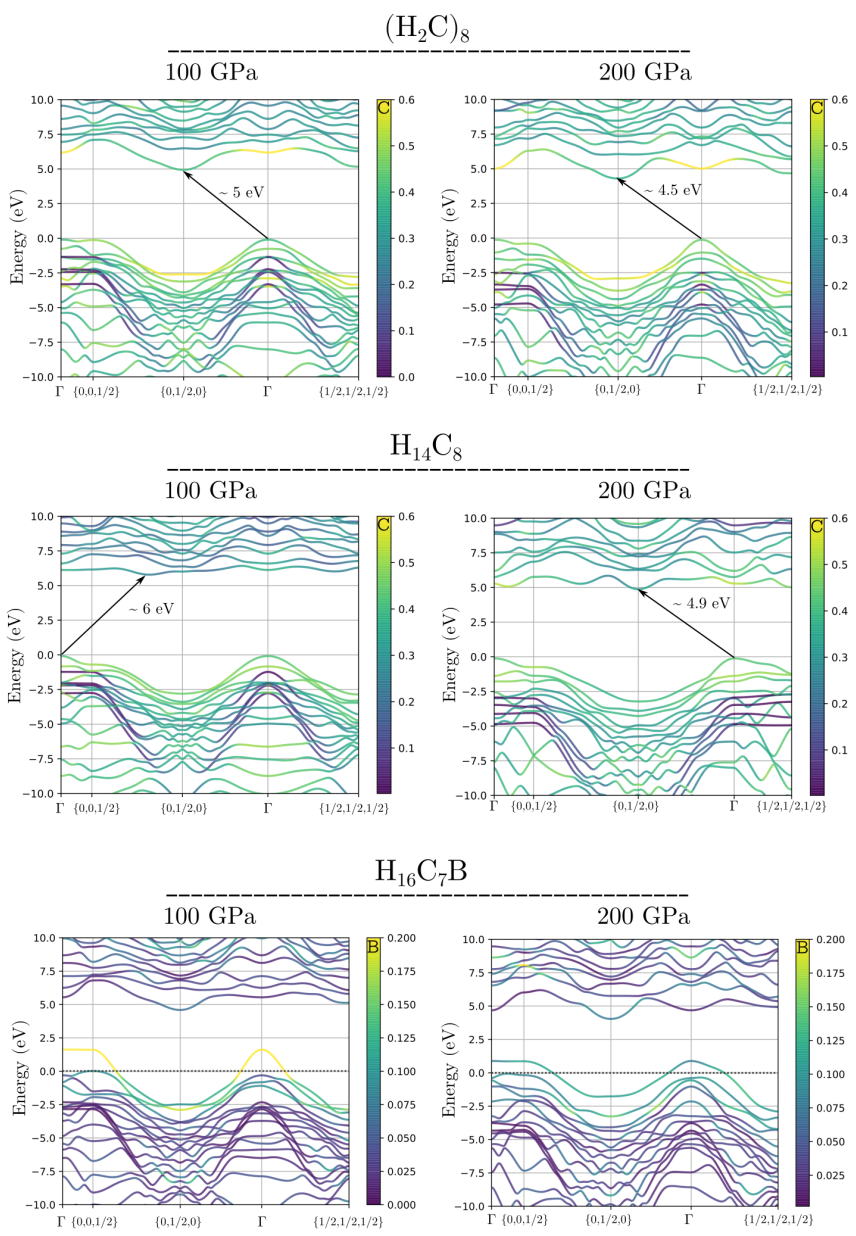

Figure 3. Electronic band structure computed for polymeric phases of polyethylene at different pressures. Polyethylene at $100 \mathrm{GPa}$ is an insulator with a indirect band gap of $5 \mathrm{eV}$ that closes to 4.5 at $200 \mathrm{GPa}$. By removing the $\mathrm{H}_{2}$ components in polyethylene the gap increases to $6 \mathrm{eV}$ at $100 \mathrm{GPa}$. Boron dope polyethylene and produces sufficient holes in the band structure that shows a metallic behavior.

$\left(\mathrm{H}_{2} \mathrm{C}\right)_{2}$ - two molecules of ethylene - in a periodic simulation box to pressures above $50 \mathrm{GPa}$.

We sample the enthalpy landscape with the minima hopping method (MHM $)^{67,68}$, using the two formula unit cells $\left(\mathrm{H}_{2} \mathrm{C}\right)_{2}$, for selected pressures in the range of 50 to $200 \mathrm{GPa}$. This method has been successfully used for global optimization of an ample number of materials ${ }^{69,70}$ and was designed to thoroughly scan the low-lying energy landscape of any compound. Within this method, stable phases are identified by performing short consecutive molecular dynamics escape steps, followed by local geometry relaxations. The enthalpy surface is efficiently mapped by aligning the velocities in initial molecular dynamics steps approximately along the soft-mode directions $^{71}$. In this way, the Bell-Evans-Polanyi ${ }^{72}$ principle is exploited to steer the search towards low energy structures.

In Fig. 1 different optimized polymeric structures are 
$50 \mathrm{GPa}$
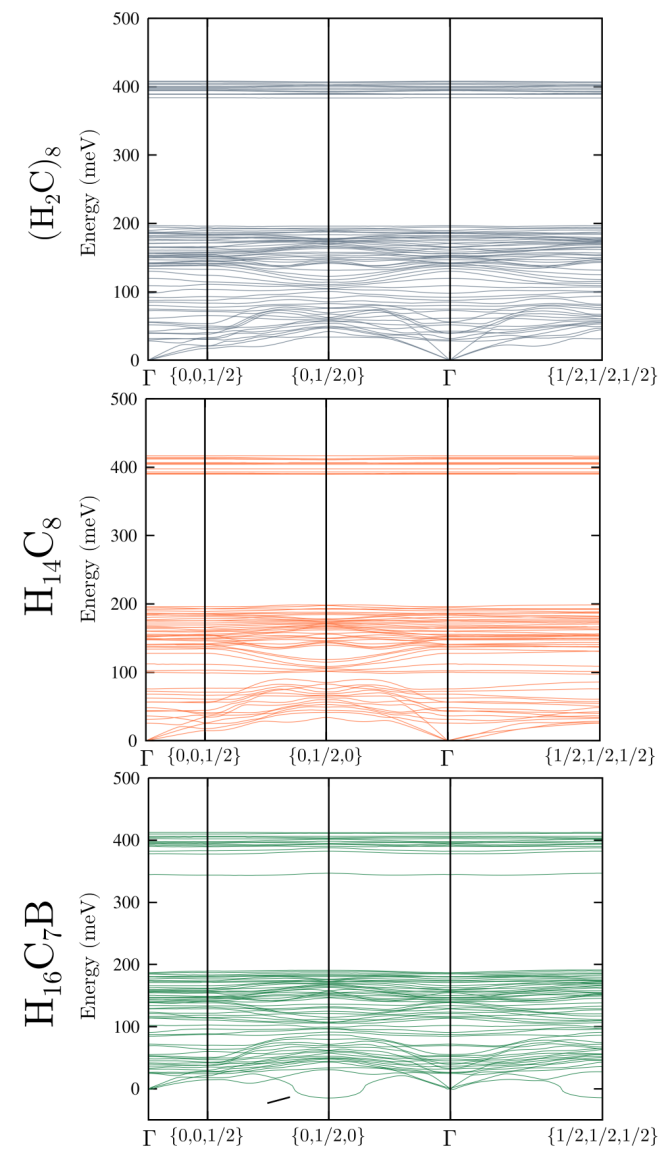

$100 \mathrm{GPa}$
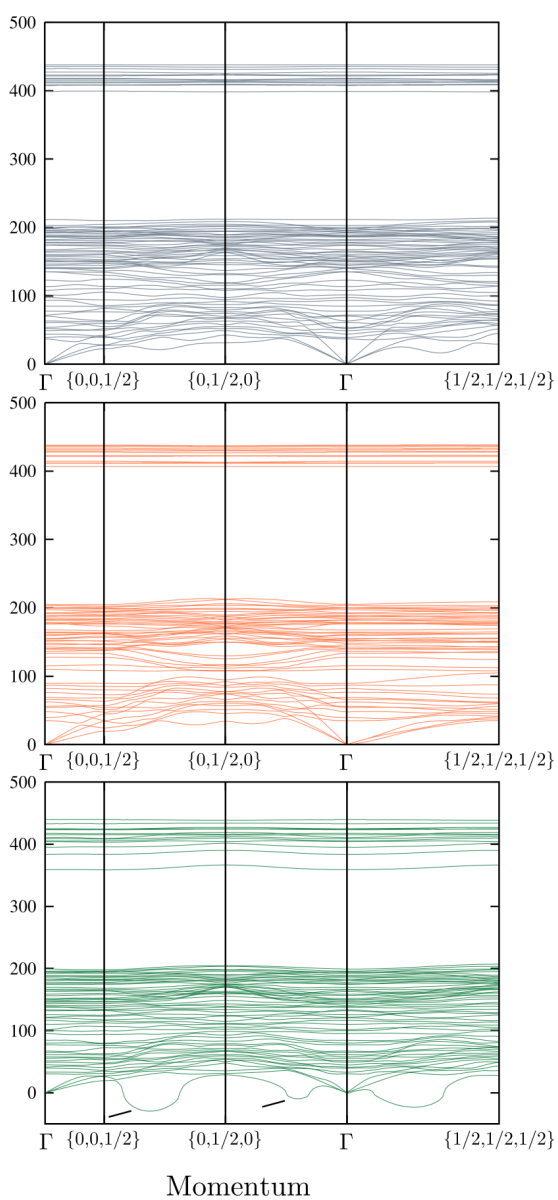

$200 \mathrm{GPa}$
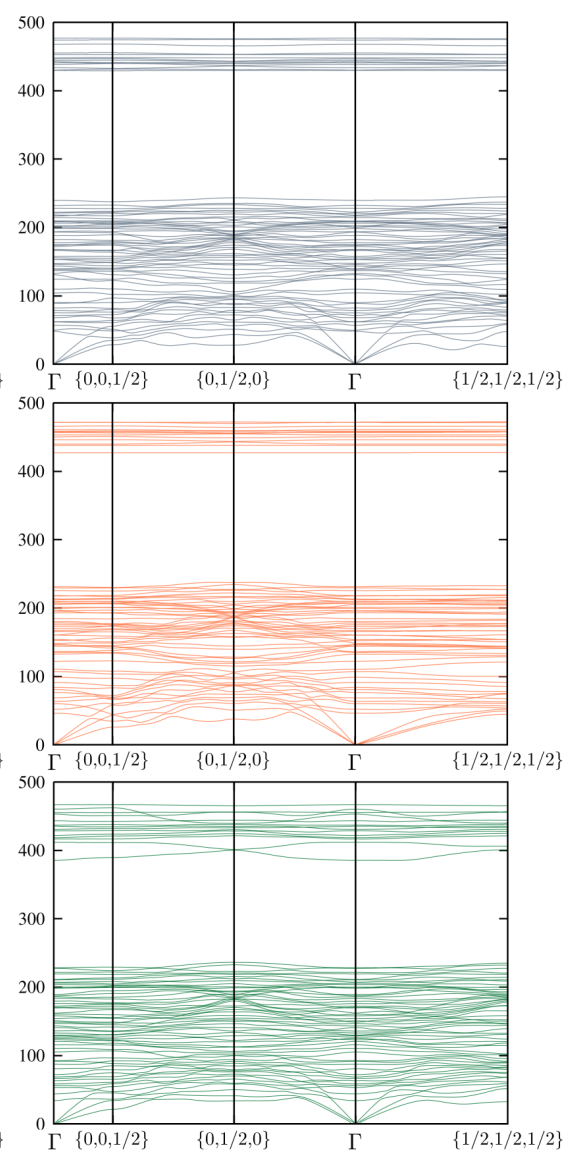

Figure 4. Phonon band dispersion computed for polymeric phases of polyethylene as a function pressure. The polymeric structures are stable in the range of pressure studied. $\mathrm{H}_{16} \mathrm{BC}_{7}$ (doped) is stable for pressures above $\sim 170$. In the contrary, $\mathrm{H}_{14} \mathrm{BC}_{7}$ (doped) is not stable and requires higher pressure for stabilization (not shown).

shown. The summary of enthalpy of formation for polymeric phases with respect to decomposition towards elemental carbon and hydrogen is shown in Fig. 2. First: the structural searches conducted for $\left(\mathrm{H}_{2} \mathrm{C}\right)_{2}$ (6 atoms cell) provide a glimpse into the complexity of the system. No stable structure, i.e. below the formation enthalpy of decomposition (zero line in Fig. 2), was found. The lowest allotrope lies almost $1.7 \mathrm{eV}$ above the stability line. The structure, consistent of intercalated polymeric $\mathrm{H}_{2} \mathrm{C}$ (note the colored circle), is shown in the top right panel of Fig. 1 along two different directions. Second: the number of atoms considered, within the relatively small cell (6 atoms), is not sufficient to conclusively rule out the possibility of thermodynamically stable polyethylene phases at high pressure. However, the complexity of the global minimum search increases exponentially with the number of atoms in the unit cell. The problem is no longer tractable even for tens of atoms when densityfunctional level of theory is used. To circumvent this issue in an efficient and timely manner, supercells of the local minima (found with small cells) were created and subsequently relaxed along soft-phonon directions. With this procedure, a novel polymeric phase, that is thermodynamically stable against elemental decomposition, was identified. The structural motif can be seen in Fig. 1. Interestingly, the new phase (top left panel marked $\left(\mathrm{H}_{2} \mathrm{C}\right)_{8}$ at $\left.200 \mathrm{GPa}\right)$ consists of two distinctive polymeric groups: polymerized cyclohexanes in "boat" configurations $^{73}$ (colored in orange) and interconnected methylene groups (colored in blue). The two units form a polymeric crystal with P1 symmetry, that lies considerably lower in the enthalpy plot (see dashed black line in Fig. 2) than the small $\left(\mathrm{H}_{2} \mathrm{C}\right)_{2}$ structure. A quick comparison with enthalpies computed by Wen-HoffmannAshcroft ${ }^{74}$ for predicted phases of polymeric benzene and layered graphane under pressure suggests that our structure is enthalpically lower. While not exact, the comparison is reasonable as the same Kohn-Sham functional (GGA-PBE) was used in their calculations and the zero reference line was computed with respect to elemental decomposition (carbon + hydrogen) into the same crystal structures as used in our work (see Fig. 10 in Ref $^{74}$ ). 
An additional notable feature of the new structure is the $\mathrm{H}_{2}$ molecules occupying voids between the two polymeric groups present in the structural motif. Hence, a logical question arises, what is the role played by the $\mathrm{H}_{2}$ molecules in the whole system? Third: to address this question the $\mathrm{H}_{2}$ components were removed from the low-enthalpy structure of polyethylene $\left(\mathrm{H}_{2} \mathrm{C}\right)_{8}$ and a geometry optimization was performed allowing all degrees of freedom to relax. The computed enthalpy is shown in Fig. 2 marked by a dashed-dot blue line. At low pressures, the stability is not strongly affected by the lack of $\mathrm{H}_{2}$ and similar enthalpies are found when compared to polyethylene. Compressing the system further, however, results in increased stabilization of the polymeric phase when $\mathrm{H}_{2}$ is removed. This is not a surprising outcome, one could simply imagine that under compression the reduction of volume brings the two main polymeric groups closer together, pushing out the $\mathrm{H}_{2}$ molecules due to repulsive Coulomb interactions (dehydrogenating the polyethylene at high pressure). The resulting rearrangements of the structural motifs, following $\mathrm{H}_{2}$ removal, can be seen in the bottom-right panel of Fig. 1. At $200 \mathrm{GPa}$ there is reduction in volume of $6 \%$ by removing the $\mathrm{H}_{2}$ components, but the overall $\mathrm{C}-\mathrm{H}$ distance in the boats (polymeric lines) and angles are preserved. The two distinct motifs are now colored in orange "cyclohexanes" boat (see IUPAC definition) and in blue "methylene" group.

\section{ELECTRONIC STRUCTURE: RISE OF METALIZATION AND SUPERCONDUCTIVITY}

The electronic band structures calculated for the different polymeric phases are summarized in Fig. 3 (only two pressure points are shown). Polyethylene at $100 \mathrm{GPa}$ is an insulator with a indirect band gap of $5 \mathrm{eV}$ that closes by $0.5 \mathrm{eV}$ at $200 \mathrm{GPa}$. Assuming a linear dependence of the band gap, pressures above $1 \mathrm{TPa}$ (tera-Pascal) would be necessary to fully close the gap. The novel phase without $\mathrm{H}_{2}$ (marked as $\mathrm{H}_{14} \mathrm{C}_{8}$ ) possesses a larger indirect band gap of $6 \mathrm{eV}$ at $100 \mathrm{GPa}$. Interestingly, the top of the conduction band shows decreased dispersion when compared to the conduction band of $\mathrm{H}_{16} \mathrm{C}_{8}$. This is due to the increase in repulsive forces between the two main polymeric chains, while in $\mathrm{H}_{16} \mathrm{C}_{8}$ these are mediated via $\mathrm{H}_{2}$ molecules. The gap closes to a similar value of $4.9 \mathrm{eV}$ at $200 \mathrm{GPa}$, but still an enormous pressure would be necessary to metallize this system.

As was previously mentioned, a path to induce metallization in an otherwise insulating system is provided by doping. In the case of $\mathrm{H}_{2} \mathrm{O}$, nitrogen was found to be the only element that could hole dope the lattice of ice-X at high pressure ${ }^{52}$. Following this idea, one could expect boron to hole dope the system under consideration in this work. Substitutions at different carbon-sites were tested (full optimization of atoms and cell) and ranked energetically. We show the lowest enthalpy structural motif of $\mathrm{H}_{16} \mathrm{BC}_{7}$ at $200 \mathrm{GPa}$ in the bottom left panel of Fig. 1, equivalent to $12 \%$ boron doping. The electronic structure is shown in the bottom panel of Fig. 3, confirming our suspicion that boron successfully hole-dopes polyethylene, in the polymeric high-pressure phase, turning it metallic. The color in the band structure plot shows the projected Kohn-Sham states corresponding to boron (normalized to the value of 0.2 for clarity), identifying the energy regions in which boron states offer strong contributions. The occupation at the Fermi level $N\left(E_{F}\right)$ is $0.65 e^{-} /$state at $100 \mathrm{GPa}$ and decreases to $0.5 e^{-} /$state at $200 \mathrm{GPa}$.

The thermodynamic stability and the possibility of metalization upon doping have been considered so far. Another, essential step to consider is the structural stability of these phases, i.e. whether all phonon frequencies are real. Fig. 4 encompasses the computed phonon dispersions along different $q$-vectors in the irreducible zone of the phases previously discussed. Three selected pressures are shown: 50, 100 and $200 \mathrm{GPa}$, top row shows the phonon dispersion of $\left(\mathrm{H}_{2} \mathrm{C}\right)_{8}$, middle row $-\mathrm{H}_{14} \mathrm{C}_{8}$ and bottom row $-\mathrm{H}_{16} \mathrm{BC}_{7}$. The analysis of the phonon density of states shows two distinct zones of vibrations: the high frequency modes purely hydrogen and $\mathrm{C}-\mathrm{H}$ vibrations up to $250 \mathrm{meV}$. The non-doped cases are all dynamically stable. The low pressure phases of $\mathrm{H}_{16} \mathrm{BC}_{7}$ are dynamically unstable (imaginary phonon frequencies) along finite $q$-vectors in the Brillouin zone (see arrows in Fig. 4).

We now turn to the discussion of superconducting properties in the doped system. It is well known that upon doping covalently bonded system may show superconducting behavior, given that the doping level is sufficiently high ${ }^{51,75-79}$. In addition to doping, compounds formed by light elements such as carbon and hydrogen are particularly promising for superconductivity due to their light masses, that correspond to high energy vibrational modes leading to increased electron-phonon coupling.

Different theoretical methods for studying the effect of doping on the superconducting properties are available. The simplest - a rigid shift of the Fermi level, leaving both Kohn-Sham eigenvalues and eigenfunctions unchanged, is computationally cheap. The drawbacks: effects of structural relaxations on electronic states are ignored and changes in the phonon spectrum induced by excess charge and the formation of a Fermi surface are not accounted for. An alternative approach is to alter the electron number in the simulations. Extra electrons are compensated by a uniform (jellium) background to ensure charge neutrality. This method properly accounts for metalization and lattice relaxations in the doped system and is physically similar to doping by an intense electrostatic field ${ }^{80}$. A third approach to simulate chemical doping. A supercell of the host material is created, allowing for site-substitutions with different elements, that can create holes or serve as electron donors ${ }^{81}$. Chemical doping is complex and cumbersome to simulate as it involves local structural relaxations of large supercells. 

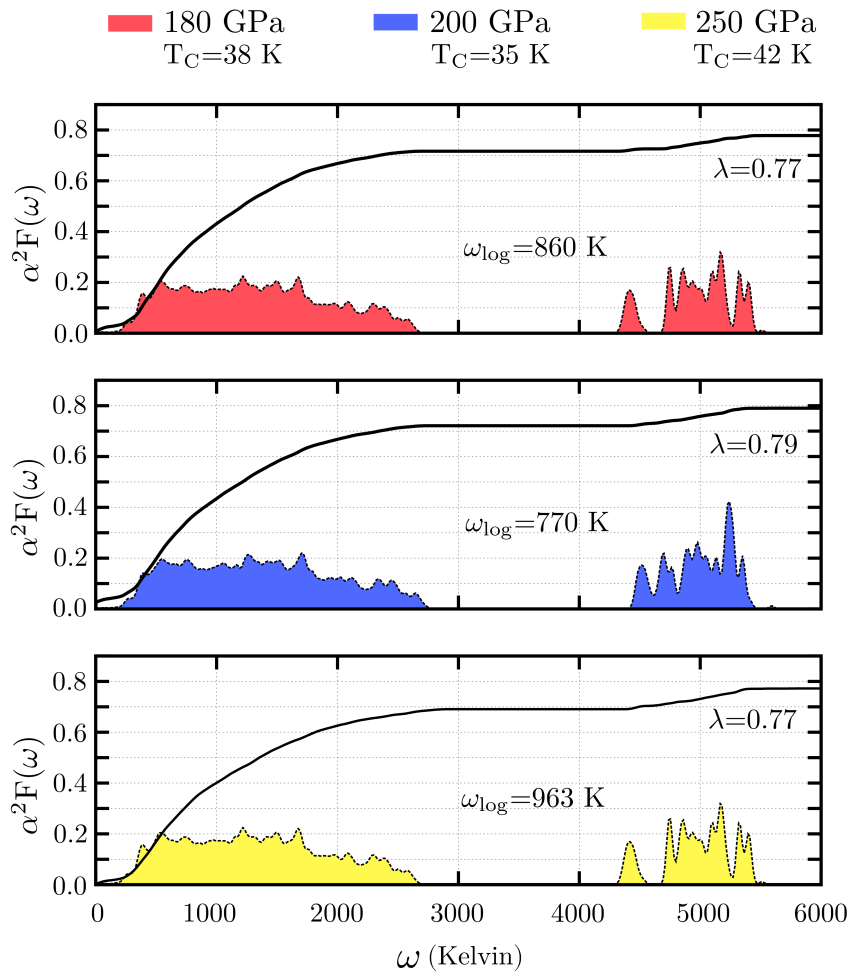

Figure 5. Eliashberg spectral function (colored areas) and integration curve of the electron-phonon coupling constant $\lambda(\omega)$ (solid line) for boron hole-doped $\left(\mathrm{H}_{2} \mathrm{C}\right)_{8}(12 \%)$ in the polymeric phase as a function of pressure. transition temperature of superconductivity are predicted for the system to range between 35 to $45 \mathrm{~K}$.

Furthermore, in order to find suitable elements different doping locations, such as inequivalent site-substitutions, interstitials, etc. has to be consider, before a prediction can be attempted.

As the two proposed structures $\left(\mathrm{H}_{2} \mathrm{C}\right)_{8}$ and $\mathrm{H}_{14} \mathrm{C}_{8}$ are dynamically stable and boron substitution, as shown in the previous section, leads to their metallization we chose the third approach in our work, i.e. calculating the superconducting properties in the chemically doped supercell $\mathrm{H}_{16} \mathrm{BC}_{7}$. It is worth mentioning, that motivated by the observation of hole-doping induced by boron a further handful of elements were chosen and investigated as dopants at low pressures. These results are shown and discussed in the Appendix.

The electron-phonon spectral function $\alpha^{2} F(\omega)$, as calculated within linear-response for the doped $\left(\mathrm{H}_{2} \mathrm{C}\right)_{8}$ polymer, is shown for different pressure in Fig. 5. In the plot, frequency is shown in the abscissa and $\alpha^{2} F(\omega)$ shown in the ordinate; the system possesses a moderate electronphonon coupling $(\lambda)$ calculated between 0.77 and 0.8 depending on the pressure. The first moments of the average phonon energy $\left(\omega_{\log }\right)$ are at $74 \mathrm{meV}(860 \mathrm{~K}), 66 \mathrm{meV}$ $(770 \mathrm{~K})$ and $83 \mathrm{meV}(963 \mathrm{~K})$ for $180 \mathrm{GPa}, 200 \mathrm{GPa}$ and $250 \mathrm{GPa}$, respectively. The calculated transition temperature $\left(\mathrm{T}_{\mathrm{C}}\right)$ is non-negligible and oscillates between values of 35 to $42 \mathrm{~K}$ in the range between $180 \mathrm{GPa}$ to $250 \mathrm{GPa}$. This estimation is calculated with McMillan parametrization $^{82}$ and a standard value $\left(\mu^{*}=0.1\right)$ for the Coulomb pseudopotential is assumed ${ }^{83,84}$. It is possible that the $\mu^{*}=0.1$ assumption becomes inapplicable in the high pressure regime. Yet, further assessments of the exact value of the Coulomb pseudopotential on polymeric low dimensional systems - are difficult to make. If instead, a very strong Coulomb repulsion was chosen, in principle, the superconducting phase could vanish completely. While using values of 0.2 for $\mu^{*}$, reduces the $T_{\mathrm{C}}$ by roughly a half: $15 \mathrm{~K}$ at $180 \mathrm{GPa}$ and $200 \mathrm{GPa}$ and $17 \mathrm{~K}$ at $250 \mathrm{~K}$. These would, nevertheless, be well measurable values of $\mathrm{T}_{\mathrm{C}}$.

\section{DISCUSSION}

Among polymers, polyethylene is by far the most common and widely produced ${ }^{85}$. Low-density polyethylene, for instance, can be obtained by compressing the ordered crystal phase of ethylene at room temperature above $3 \mathrm{GPa}$ with a continuous-wave laser uses as an optical catalyst ${ }^{65}$. Highly crystalline polymers can be similarly obtained. For pressures up to $\sim 40 \mathrm{GPa}$, polyethylene has been subject to detailed experimental ${ }^{85,86}$ and theoretical studies ${ }^{87}$. The structural sequence of transformations of polyethylene at relatively low pressures is well documented. Indeed, it was recently shown that the ambient structural properties of polyethylene can be fully recovered after sequential compression/decompression cycles, indicating that polymers were structurally and chemically stable at least up to $50 \mathrm{GPa}^{88}$.

No experimental or theoretical work reporting on the high pressure domain above $50 \mathrm{GPa}$ exists. Interestingly, the two polymeric structures predicted in this work are considerably lower in enthalpy than those studied by Fontana et al. ${ }^{87}$ (Pnam, $A 2 / m$ and $\mathrm{P} 2_{1} / \mathrm{m}$ phases at $40 \mathrm{GPa})$ and the same two phases $\left(\mathrm{H}_{2} \mathrm{C}\right)_{8}$ and $\mathrm{H}_{14} \mathrm{C}_{8}$ are comparable (or lower) in enthalpy than benzene polymorphs and graphane phases at the same pressures ${ }^{74}$. In this context, we call for experimental studies at pressures above $50 \mathrm{GPa}$ to verify our findings: is the predicted polymeric phase the product of compressed polyethylene/benzene? Our theoretical calculations point towards a low-symmetry phase comprised of $\mathrm{H}_{6} \mathrm{C}_{4}$ and $\mathrm{H}_{2} \mathrm{C}$ groups stabilized by interstitial $\mathrm{H}_{2}$ molecules, while further compression may result in the release of the $\mathrm{H}_{2}$ from the main host.

Metalization of polyethylene by boron doping was investigated in the study. The range of stability identified for the boron doped $\left(\mathrm{H}_{16} \mathrm{BC}_{7}\right)$ phase is relatively narrow. Dynamical stability is observed in the $170 \mathrm{GPa}$ to $250 \mathrm{GPa}$ range, while enthalpically elemental decomposition is favored above $200 \mathrm{GPa}$. This limits the range of stability and possible synthesis to $\sim 30 \mathrm{GPa}$ window. How can doping be achieved at high pressure? This question is of complex nature and non-trivial to address even 
for the most experienced high-pressure experimentalists. One could hypothesize a laser-heating aided decomposition of molecules that releases agents (doping elements) which target native vacancies or incorporate as interstitials at high pressure. A somewhat "simpler problem" should then be addressed: how can control over vacancies, or formation of native defects, be achieved under high pressure? The evolution of crystalline defects under pressure is a field with many open questions that still remains poorly studied.

In conclusion, we two crystalline structures of polyethylene occurring at pressures above $50 \mathrm{GPa}$ were predicted. These phases are likely to be accessible by experimental methods for verification. Finally, we have also shown the possibility of inducing metallicity and superconductivity with a $\mathrm{T}_{\mathrm{C}}$ of $\sim 35 \mathrm{~K}$ by doping the insulating organic-polymeric polyethylene under pressure.

\section{COMPUTATIONAL METHODS}

Energy, atomic forces and stresses were evaluated at the density functional theory level with the Perdew-Burke-Erzernhof (PBE) ${ }^{89}$ parametrization of the exchange-correlation functional. A plane wave basis-set cutoff energy of $900 \mathrm{eV}$ was used to expand the wavefunction together with the projector augmented wave (PAW) method, as implemented in the Vienna Ab Initio Simulation Package VASP ${ }^{90}$. Reciprocal space $k$-grids were generated with the Monhorst-Pack method with spacing of 0.3. Supercell structures were constructed using CIF2CELL code ${ }^{91}$. Geometry relaxations were performed with tight parameters such that the forces acting on the atoms were below $2 \mathrm{meV} / \AA$ and the stresses were less than $0.1 \mathrm{eV} / \AA^{3}$.

The phonon spectra and the electron-phonon matrix elements were calculated with density functional perturbation theory ${ }^{92,93}$, as implemented in the plane-wave based code ABINIT ${ }^{94}$. Core atomic states were described in the norm-conserving pseudo-potential approximation; valence state were described by a plane-wave basis set with an energy cutoff of $1100 \mathrm{eV}$.

For electron-phonon matrix calculations regular $\Gamma$ centered $k=8 \times 8 \times 8$ and $q=4 \times 4 \times 4$ meshes were used. The electron-phonon coupling at the Fermi energy is described in the isotropic approximation by the Eliashberg spectral functions ${ }^{83}$, defined as:

$$
\alpha^{2} F(\omega)=\frac{1}{N_{E_{F}}} \sum_{\mathbf{k q}, \nu}\left|g_{\mathbf{k}, \mathbf{k}+\mathbf{q}, \nu}\right|^{2} \delta\left(\epsilon_{\mathbf{k}}\right) \delta\left(\epsilon_{\mathbf{k}+\mathbf{q}}\right) \delta\left(\omega-\omega_{\mathbf{q}, \nu}\right)
$$

where $N_{E_{F}}$ is the DOS at the Fermi level, $\omega_{\mathbf{q}, \nu}$ is the phonon frequency of mode $\nu$ at wavevector $\mathbf{q}$ and $\left|g_{\mathbf{k}, \mathbf{k}+\mathbf{q}, \nu}\right|$ is the electron-phonon matrix element between two electronic states with momenta $\mathbf{k}$ and $\mathbf{k}+\mathbf{q}$. All computed $\alpha^{2} F(\omega)$ are collected in Fig. 5. Anisotropy effects have been estimated to be irrelevant in the calculation of $\mathrm{T}_{\mathrm{C}}$ and are neglected in this work. Two significant moments of the Eliashberg function $\lambda$ and $\omega_{\log }$, defined as:

$$
\begin{aligned}
\lambda & =2 \int \frac{\alpha^{2} F(\omega)}{\omega} d \omega \\
\omega_{\log } & =\exp \left[\frac{2}{\lambda} \int \alpha^{2} F(\omega) \frac{\ln (\omega)}{\omega} d \omega\right]
\end{aligned}
$$

express, respectively, the electron-phonon coupling and the effective phononic energy.

Finally, the reference structures for solid hydrogen were taken from Ref. ${ }^{95}$ and are $P 6_{3} m(0-120 \mathrm{GPa})$ and $C 2 / c(120-200 \mathrm{GPa})$. Cubic structure of diamond $F d-3 m$ is the most stable allotrope in the $50-200 \mathrm{GPa}$ range. These structure were used to calculate the zero line for elemental decomposition.

\section{ACKNOWLEDGMENTS}

The authors acknowledge the hospitality of the cini-II meeting held in Cala Sinzias, where part of this work was written. The authors would like to thank Dr. Daniele Tomerini for helpful ideas and insightful discussion into the chemistry of these systems. Without the vast computational resources under the project s752 from the Swiss National Supercomputing Center (CSCS) in Lugano, this work would not have been possible. These resources are greatly acknowledged. This work was partially funded by MARVEL.

\section{APPENDIX}

Ten different elements (Li, Be, Na, B, N, Al, Si, Ga, P, S) were tested as possible substitutional dopants at the carbon site. The study was conducted at low pressure $(50 \mathrm{GPa})$ for a single carbon site belonging to the main polymeric chain of $\mathrm{H}_{6} \mathrm{C}_{4}$ (shown in orange in Fig. 1). In this pre-screening step we were interested only in elements that do not strongly disrupt the local environment (i.e. modify the polymeric chains). Fig. 6 shows the elements which we found to preserve the overall structural motif of polyethylene. The elements ( $\mathrm{Li}, \mathrm{Na}, \mathrm{Be}, \mathrm{Al}$, P) that are not shown significantly alter the structure of polyethylene and produce dimerization of the polymeric chains that suggests amorphization.

Interestingly, nitrogen substitution induces a distortion, that leads to the formation of $\mathrm{H}_{2}$ chains along the polymeric lines. This resembles the linear chains of hydrogen seen in $\mathrm{H}_{3} \mathrm{~S}$ and may be an interesting avenue to explore in the future. Silicon, since it is isoelectronic to carbon, does not dope the system. However, volume effects result in a drastic reduction of the electronic gap from $6 \mathrm{eV}$ to $2.5 \mathrm{eV}$. Sulfur and gallium lead to volume expansion and produce strong hybridization in the electronic bands, that heavily modify the electronic structure. The highly dispersive band found below the Fermi 

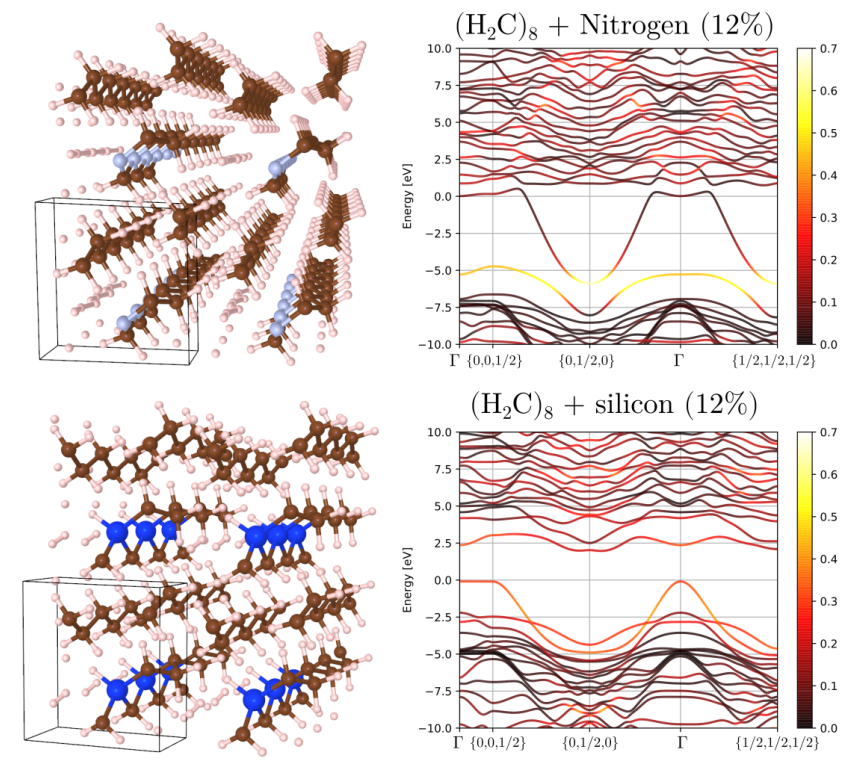

$\left(\mathrm{H}_{2} \mathrm{C}\right)_{8}+$ silicon $(12 \%)$
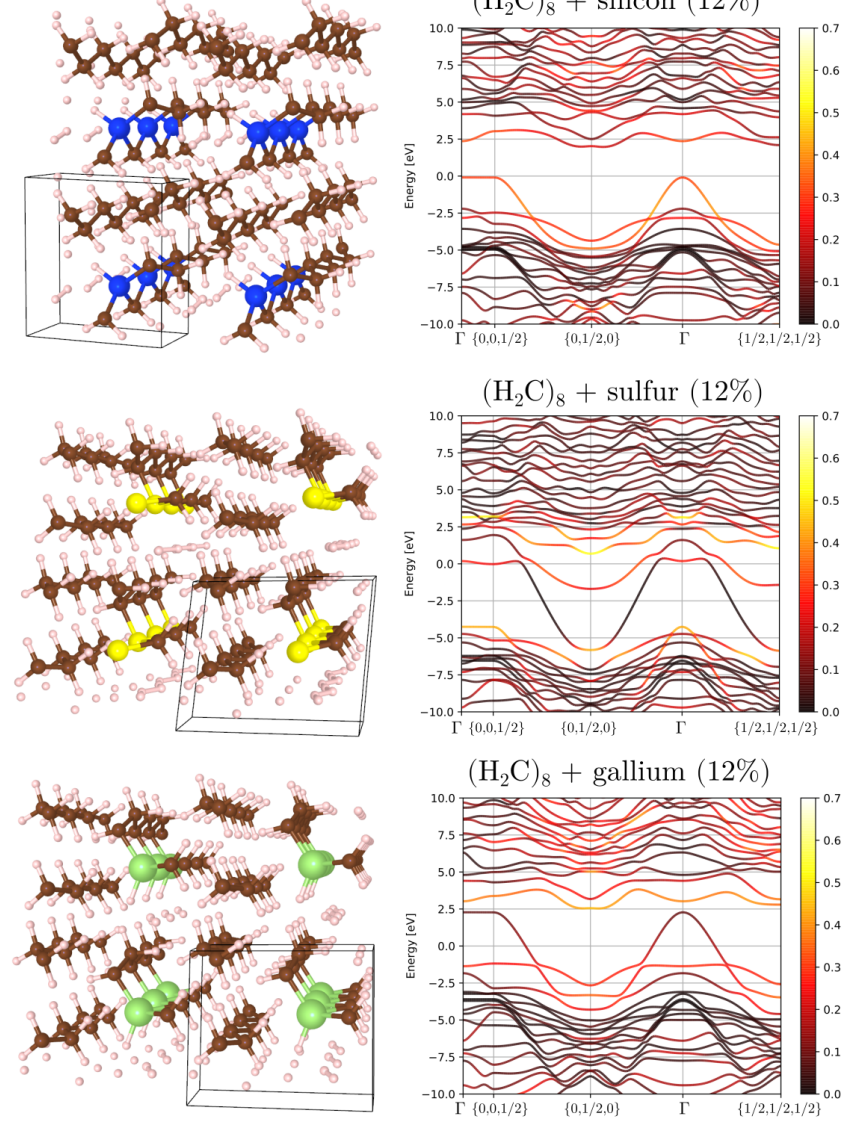

$\left(\mathrm{H}_{2} \mathrm{C}\right)_{8}+$ gallium $(12 \%)$

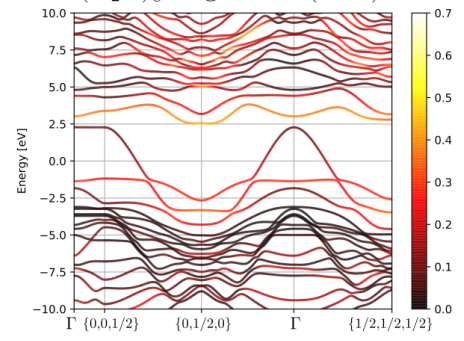

Figure 6. Left panels: crystal structure of $\left(\mathrm{H}_{2} \mathrm{C}\right)_{8}$ doped with N, Si, S and Ga. Right panels: shows the corresponding electronic band structure with Kohn-Sham projected states. level $(0$ to $-5 \mathrm{eV})$ with $\mathrm{N}, \mathrm{S}$ and $\mathrm{Ga}$ doping, arises from the $\mathrm{H}_{2}$ molecule/chain states.
1 P. W. Bridgman, The Nobel Prize in Physics 1946 -, (1946).

2 M. Eremets, High pressure experimental methods (Oxford University Press, 1996).

3 P. F. McMillan, Nature materials 4, 715 (2005).

${ }^{4}$ E. Wigner and H. á. Huntington, The Journal of Chemical Physics 3, 764 (1935).

5 N. W. Ashcroft, Phys. Rev. Lett. 92, 187002 (2004).

${ }^{6}$ M. I. Eremets, I. A. Trojan, S. A. Medvedev, J. S. Tse, and Y. Yao, Science 319, 1506 (2008).

7 X.-J. Chen, V. V. Struzhkin, Y. Song, A. F. Goncharov, M. Ahart, Z. Liu, H.-k. Mao, and R. J. Hemley, Proceedings of the National Academy of Sciences 105, 20 (2008).

8 D. Y. Kim, R. H. Scheicher, S. LebÃ šgue, J. Prasongkit,
B. Arnaud, M. Alouani, and R. Ahuja, Proceedings of the National Academy of Sciences 105, 16454 (2008).

9 S. Wang, H.-k. Mao, X.-J. Chen, and W. L. Mao, Proceedings of the National Academy of Sciences 106, 14763 (2009).

10 M. Hanfland, J. E. Proctor, C. L. Guillaume, O. Degtyareva, and E. Gregoryanz, Phy. Rev. Lett. 106, 095503 (2011).

11 D. Y. Kim, R. H. Scheicher, C. J. Pickard, R. J. Needs, and R. Ahuja, Phys. Rev. Lett. 107, 117002 (2011).

12 J. A. Flores-Livas, M. Amsler, T. J. Lenosky, L. Lehtovaara, S. Botti, M. A. L. Marques, and S. Goedecker, Phys. Rev. Lett. 108, 117004 (2012). 
13 D. Duan, Y. Liu, F. Tian, D. Li, X. Huang, Z. Zhao, H. Yu, B. Liu, W. Tian, and T. Cui, Sci. Rep. 4 (2014), http://dx.doi.org/10.1038/srep06968.

14 A. P. Drozdov, M. I. Eremets, I. A. Troyan, V. Ksenofontov, and S. I. Shylin, Nature 525, 2015/08/17/online (2015).

15 I. Troyan, A. Gavriliuk, R. Rüffer, A. Chumakov, A. Mironovich, I. Lyubutin, D. Perekalin, A. P. Drozdov, and M. I. Eremets, Science 351, 1303 (2016).

16 M. Einaga, M. Sakata, T. Ishikawa, K. Shimizu, M. I. Eremets, A. P. Drozdov, I. A. Troyan, N. Hirao, and Y. Ohishi, Nature Physics (2016).

17 I. Errea, M. Calandra, C. J. Pickard, J. Nelson, R. J. Needs, Y. Li, H. Liu, Y. Zhang, Y. Ma, and F. Mauri, Phys. Rev. Lett. 114, 157004 (2015).

18 C. Heil and L. Boeri, Phys. Rev. B 92, 060508 (2015).

19 R. Akashi, W. Sano, R. Arita, and S. Tsuneyuki, Phys. Rev. Lett. 117, 075503 (2016).

20 A. J. Flores-Livas, A. Sanna, and E. Gross, Eur. Phys. J. B 89, 1 (2016).

21 J. A. Flores-Livas, A. Sanna, and S. Goedecker, Novel Superconducting Materials 3, 6 (2017).

22 A. Drozdov, M. Eremets, and I. Troyan, arXiv preprint arXiv:1508.06224 (2015).

23 J. A. Flores-Livas, M. Amsler, C. Heil, A. Sanna, L. Boeri, G. Profeta, C. Wolverton, S. Goedecker, and E. K. U. Gross, Phys. Rev. B 93, 020508 (2016).

${ }^{24}$ V. V. Struzhkin, M. I. Eremets, W. Gan, H.-k. Mao, and R. J. Hemley, Science 298, 1213 (2002).

25 Y. Li, G. Gao, Y. Xie, Y. Ma, T. Cui, and G. Zou, Proceedings of the National Academy of Sciences 107, 15708 (2010).

${ }^{26}$ K. Shimizu, K. Amaya, and N. Suzuki, Journal of the Physical Society of Japan 74, 1345 (2005), http://dx.doi.org/10.1143/JPSJ.74.1345.

27 G. Profeta, C. Franchini, N. Lathiotakis, A. Floris, A. Sanna, M. A. L. Marques, M. Lüders, S. Massidda, E. K. U. Gross, and A. Continenza, Phys. Rev. Lett. 96, 047003 (2006).

28 J. A. Flores-Livas, A. Sanna, A. P. Drozdov, L. Boeri, G. Profeta, M. Eremets, and S. Goedecker, Physical Review Materials 1, 024802 (2017).

29 J. Flores Livas, Computational and experimental studies of sp3-materials at high pressure, Ph.D. thesis, Lyon 1 (2012).

${ }^{30}$ W. Grochala, R. Hoffmann, J. Feng, and N. W. Ashcroft, Angewandte Chemie International Edition 46, 3620 (2007).

31 O. Degtyareva, J. E. Proctor, C. L. Guillaume, E. Gregoryanz, and M. Hanfland, Solid State Communications 149, 1583 (2009).

32 C. F. Richardson and N. W. Ashcroft, Phys. Rev. Lett. 78, 118 (1997).

33 P. Cudazzo, G. Profeta, A. Sanna, A. Floris, A. Continenza, S. Massidda, and E. K. U. Gross, Phys. Rev. Lett. 100, 257001 (2008).

${ }^{34}$ P. Cudazzo, G. Profeta, A. Sanna, A. Floris, A. Continenza, S. Massidda, and E. K. U. Gross, Phys. Rev. B 81, 134505 (2010).

35 P. Cudazzo, G. Profeta, A. Sanna, A. Floris, A. Continenza, S. Massidda, and E. K. U. Gross, Phys. Rev. B 81, 134506 (2010).

36 M. I. Eremets and I. A. Troyan, Nat. Mat. 10, 927 (2011).

37 P. Loubeyre, F. Occelli, and R. LeToullec, Nature 416, 13 (2002).
38 I. I. Naumov and R. J. Hemley, Accounts of Chemical Research 47, 3551 (2014), pMID: 25369180, http://dx.doi.org/10.1021/ar5002654.

39 C.-S. Zha, Z. Liu, and R. Hemley, Phys. Rev. Lett. 108, $146402(2012)$.

40 X.-D. Liu, P. Dalladay-Simpson, R. T. Howie, B. Li, and E. Gregoryanz, Science 357, eaan2286 (2017).

41 R. P. Dias and I. F. Silvera, Science (2017), 10.1126/science.aal1579.

42 M. Eremets, I. Troyan, and A. Drozdov, arXiv preprint arXiv:1601.04479 (2016).

43 M. Zaghoo, A. Salamat, and I. F. Silvera, Physical Review B 93, 155128 (2016).

44 A. L. Ruoff, H. Luo, and Y. K. Vohra, Journal of applied physics 69, 6413 (1991).

45 E. Gamboa, L. Fletcher, H. Lee, M. MacDonald, U. Zastrau, M. Gauthier, D. Gericke, J. Vorberger, E. Granados, J. Hastings, et al., Band gap opening in strongly compressed diamond observed by $x$-ray energy loss spectroscopy, Tech. Rep. (SLAC National Accelerator Laboratory (SLAC), Menlo Park, CA (United States), 2016).

46 R. J. Hemley, High Pressure Research 30, 581 (2010).

47 L. Zhang, Y. Wang, J. Lv, and Y. Ma, Nature Reviews Materials 2, 17005 (2017).

48 E. A. Ekimov, V. A. Sidorov, E. D. Bauer, N. N. Mel'nik, N. J. Curro, J. D. Thompson, and S. M. Stishov, Nature 428, 542 (2004).

49 E. Bustarret, C. Marcenat, P. Achatz, J. Kačmarčik, F. Lévy, A. Huxley, L. Ortéga, E. Bourgeois, X. Blase, D. Débarre, et al., Nature 444, 465 (2006).

50 T. Herrmannsdörfer, V. Heera, O. Ignatchik, M. Uhlarz, A. Mücklich, M. Posselt, H. Reuther, B. Schmidt, K.-H. Heinig, W. Skorupa, M. Voelskow, C. Wündisch, R. Skrotzki, M. Helm, and J. Wosnitza, Phys. Rev. Lett. 102, 217003 (2009).

51 G. Savini, A. C. Ferrari, and F. Giustino, Phys. Rev. Lett. 105, 037002 (2010).

52 J. A. Flores-Livas, A. Sanna, M. Grauzinyte, A. Davydov, S. Goedecker, and M. A. L. Marques, Scientific Reports 7, 6825 (2017).

53 G. R. Stewart, Rev. Mod. Phys. 83, 1589 (2011).

54 H. Hosono, A. Yamamoto, H. Hiramatsu, and Y. Ma, Materials Today 21, 278 (2018).

55 J. A. Flores-Livas, R. Debord, S. Botti, A. San Miguel, S. Pailhès, and M. A. L. Marques, Phys. Rev. B 84, 184503 (2011).

56 J. A. Flores-Livas, R. Debord, S. Botti, A. San Miguel, M. A. L. Marques, and S. Pailhès, Phys. Rev. Lett. 106, 087002 (2011).

57 P. A. Lee, N. Nagaosa, and X.-G. Wen, Rev. Mod. Phys. 78, 17 (2006).

58 Y. Cao, V. Fatemi, S. Fang, K. Watanabe, T. Taniguchi, E. Kaxiras, and P. Jarillo-Herrero, Nature (2018).

59 A. Sanna, C. Franchini, A. Floris, G. Profeta, N. N. Lathiotakis, M. Lüders, M. A. L. Marques, E. K. U. Gross, A. Continenza, and S. Massidda, Phys. Rev. B 73, 144512 (2006).

60 J. A. Flores-Livas and A. Sanna, Phys. Rev. B 91, 054508 (2015).

61 A. Sanna, J. A. Flores-Livas, A. Davydov, G. Profeta, K. Dewhurst, S. Sharma, and E. Gross, Journal of the Physical Society of Japan 87, 041012 (2018).

62 M. Thiery and J. Leger, The Journal of chemical physics 89, 4255 (1988). 
${ }^{63}$ L. Ciabini, F. A. Gorelli, M. Santoro, R. Bini, V. Schettino, and M. Mezouar, Physical Review B 72, 094108 (2005).

${ }^{64}$ L. Ciabini, M. Santoro, F. A. Gorelli, R. Bini, V. Schettino, and S. Raugei, Nature materials 6, 39 (2007).

65 D. Chelazzi, M. Ceppatelli, M. Santoro, R. Bini, and V. Schettino, Nature materials 3, 470 (2004).

${ }^{66}$ Y. Wang, L. Wang, H. Zheng, K. Li, M. Andrzejewski, T. Hattori, A. Sano-Furukawa, A. Katrusiak, Y. Meng, F. Liao, et al., The Journal of Physical Chemistry C 120, 29510 (2016).

67 S. Goedecker, The Journal of Chemical Physics 120, 9911 (2004).

68 M. Amsler and S. Goedecker, The Journal of Chemical Physics 133, 224104 (2010).

${ }^{69}$ M. Amsler, J. A. Flores-Livas, L. Lehtovaara, F. Balima, S. A. Ghasemi, D. Machon, S. Pailhes, A. Willand, D. Caliste, S. Botti, et al., Physical review letters 108, 065501 (2012).

70 M. Amsler, J. A. Flores-Livas, T. D. Huan, S. Botti, M. A. Marques, and S. Goedecker, Physical review letters 108, 205505 (2012).

71 M. Sicher, S. Mohr, and S. Goedecker, The Journal of Chemical Physics 134, 044106 (2011).

${ }^{72}$ F. Jensen, Introduction to Computational Chemistry: Second Edition (JW, 2011).

73 A. D. McNaught and A. D. McNaught, Compendium of chemical terminology, Vol. 1669 (Blackwell Science Oxford, 1997).

74 X.-D. Wen, R. Hoffmann, and N. Ashcroft, Journal of the American Chemical Society 133, 9023 (2011).

75 L. Boeri, J. Kortus, and O. K. Andersen, Phys. Rev. Lett. 93, 237002 (2004).

${ }^{76}$ D. Hapiuk, M. Marques, P. Mélinon, S. Botti, B. Masenelli, and J. Flores-Livas, New Journal of Physics 17, 043034 (2015).

77 D. Hapiuk, M. A. Marques, P. Melinon, J. A. Flores-Livas, S. Botti, and B. Masenelli, Physical review letters 108, 115903 (2012).

78 A. Sanna, A. V. Fedorov, N. I. Verbitskiy, J. Fink, C. Krellner, L. Petaccia, A. Chikina, D. Y. Usachov, A. Grüneis, and G. Profeta, 2D Materials 3, 025031 (2016).

79 G. Profeta, M. Calandra, and F. Mauri, Nature Phys. 8, 131 (2012).

${ }^{80}$ K. Ueno, S. Nakamura, H. Shimotani, H. T. Yuan,
N. Kimura, T. Nojima, H. Aoki, Y. Iwasa, and M. Kawasaki, Nature Nanotechnology 6, 408 EP (2011).

81 M. Grauzİinyteİ, S. Goedecker, and J. A. Flores-Livas, Chemistry of Materials 29, 10095 (2017).

82 W. L. McMillan, Phys. Rev. 167, 331 (1968).

83 P. B. Allen and B. Mitrović, Theory of Superconducting Tc, Solid State Physics, Vol. 37 (Academic Press, 1983) pp. $1-92$.

84 P. Morel and P. W. Anderson, Phys. Rev. 125, 1263 (1962).

85 D. Bassett, in Developments in crystalline polymersâ1 (Springer, 1982) pp. 115-150.

86 M. Yasuniwa, R. Enoshita, and T. Takemura, Japanese Journal of Applied Physics 15, 1421 (1976).

87 L. Fontana, D. Q. Vinh, M. Santoro, S. Scandolo, F. Gorelli, R. Bini, and M. Hanfland, Physical Review B 75, 174112 (2007).

88 L. Fontana, M. Santoro, R. Bini, D. Q. Vinh, and S. Scandolo, The Journal of chemical physics 133, 204502 (2010).

89 J. P. Perdew, K. Burke, and M. Ernzerhof, Phys. Rev. Lett. 77, 3865 (1996).

90 G. Kresse and J. Furthmüller, Comput. Mat. Sci. 6, 15 (1996).

91 T. Björkman, Computer Physics Communications 182, 1183 (2011).

92 S. Baroni, P. Giannozzi, and A. Testa, Phys. Rev. Lett. 58, 1861 (1987).

93 X. Gonze and J.-P. Vigneron, Phys. Rev. B 39, 13120 (1989).

94 X. Gonze, F. Jollet, F. A. Araujo, D. Adams, B. Amadon, T. Applencourt, C. Audouze, J.-M. Beuken, J. Bieder, A. Bokhanchuk, E. Bousquet, F. Bruneval, D. Caliste, M. Côté, F. Dahm, F. D. Pieve, M. Delaveau, M. D. Gennaro, B. Dorado, C. Espejo, G. Geneste, L. Genovese, A. Gerossier, M. Giantomassi, Y. Gillet, D. Hamann, L. He, G. Jomard, J. L. Janssen, S. L. Roux, A. Levitt, A. Lherbier, F. Liu, I. Lukacevic, A. Martin, C. Martins, M. Oliveira, S. Poncé, Y. Pouillon, T. Rangel, G.-M. Rignanese, A. Romero, B. Rousseau, O. Rubel, A. Shukri, M. Stankovski, M. Torrent, M. V. Setten, B. V. troeye, M. Verstraete, D. Waroquier, J. Wiktor, B. Xue, A. Zhou, and J. Zwanziger, Computer Physics Communications 205, 106 (2016).

95 C. J. Pickard and R. J. Needs, Nat Phys 3, 473 (2007). 